\title{
Calibration of Surface Plasmon Resonance Imager for Biochemical Detection
}

\author{
T. Ktari, H. Baccar, M. B. Mejri, and A. Abdelghani \\ Nanotechnology Laboratory, INSAT, Carthage University, Centre Urbain Nord, 1080 Charguia Cedex, Tunisia \\ Correspondence should be addressed to A. Abdelghani, aabdelghan@yahoo.fr
}

Received 13 July 2011; Revised 28 August 2011; Accepted 4 September 2011

Academic Editor: Ulku Anik

Copyright ( 2012 T. Ktari et al. This is an open access article distributed under the Creative Commons Attribution License, which permits unrestricted use, distribution, and reproduction in any medium, provided the original work is properly cited.

\begin{abstract}
We present a new Surface Plasmon Resonance imager (SPRi) based on immobilized T4-phage for bacteria detection. First, we present the sensitivity of the SPR imager towards refractive index variation for biosensor application. The SPR imager can be calibrated versus different percentage of triethylene glycol mixture in ultrapure water. The system can be used as a refractometer with sensitivity below $5 \times 10^{-5}$ in the range of 1.33300-1.34360. Second, bacteriophage (T4-phage) can be physisorbed on gold microarray spots for bacteria detection. The kinetic physisorption of different concentrations of T4-phages can be observed in real time. Finally, two types of bacteria such as E. coli (gram negative) and Lactobacillus (gram positive) were used for positive and negative tests. The results show a selectivity of T4-phage toward E. coli with a detection limit below $10^{4} \mathrm{CFU} / \mathrm{mL}$ and with good reproducibility.
\end{abstract}

\section{Introduction}

Nowadays, sensors become an increasingly exploited technology for detection and analysis of various chemical and biological compounds in important areas such as environmental monitoring, clinical applications, security, and food safety. Among them, surface plasmon resonance (SPR) for biological applications has attracted significant interest in recent years. Studies have demonstrated the detection of biologically relevant analytes, including among others prostatespecific antigen [1-4], C-reactive protein [5], human ferritin [6], a number of DNA target sequences $[7,8]$, and different types of bacteria [9-11]. SPR is an electromagnetic wave propagating along a metal/dielectric interface. This resonant phenomenon depends on the wavelength of the excitation light, the refractive index of the metallic layer, and the dielectric interface located close to the metallic layer [1113]. A variation of the refractive index of the dielectric interface induces a variation of the resonance condition. This allows the development of optical sensors designed to detect/differentiate between samples exhibiting different refractive index, or monitor biomolecule attachment onto the transducer surface [14]. In the case of SPR imaging
(SPRi), the whole chip is literally visualized using a CCD video camera [15]. Simultaneously, the equipment monitors the changes in light reflectivity over the whole surface at a fixed resonance angle and in real time. The local changes at the surface of the chip recorded as changes of refractive index provide detailed information on any interaction of kinetic process taking place. This makes possible multiplexed label-free detection using sensors in an array format, greatly improving experimental throughput and robustness compared to classical SPR, and favoring its use in many biological applications [16, 17].

In this work, we present a new Surface Plasmon Resonance imager (SPRi) based on immobilized T4-phage on gold microarrays spots for bacteria detection. First, we study the sensitivity of the SPR imaging system towards refractive index variation. With this purpose, the SPR imager has been first calibrated versus different percentages of triethylene glycol diluted in ultrapure water. Next, we use the SPR imager for bacteria detection with T4-phage as bioreceptor. Bacteriophage (T4-phage) can be physisorbed on gold microarrays spots. The kinetic deposition of different concentrations of T4-phages has been monitored in real time with the SPRi imager. Finally, two types of bacteria, E. coli 
(gram negative) and Lactobacillus (gram positive), have been assayed as positive and negative controls, respectively, using the T4-phage-modified SPRi chips $[11,18]$. A detection limit below $10^{4} \mathrm{CFU} / \mathrm{mL}$ can be obtained.

\section{Experimental}

2.1. Reagents and Apparatus. The used bacteria were E. coli K12 (gram negative) and Lactobacillus fermentum (gram positive) diluted in PBS $(\mathrm{pH}=7.2)$ and concentration stability was assessed by monitoring the suspensions optical density (OD650) over time. The same procedure for phage storing and growing. The phosphate buffer saline solution (PBS) contained $140 \mathrm{mM} \mathrm{NaCl}, 2.7 \mathrm{mM} \mathrm{KCl}, 0.1 \mathrm{mM} \mathrm{Na}_{2} \mathrm{HPO}_{4}$, and $1.8 \mathrm{mM} \mathrm{KH}_{2} \mathrm{PO}_{4}$, adjusted at $\mathrm{pH} 7.2$. All reagents were of analytical grade and ultrapure water (resistance $\geq$ $18.2 \mathrm{M} \Omega \mathrm{cm}^{-1}$ ), produced using a Millipore Milli-Q system, was used throughout. The PBS and the ultrapure water used in this work were sterilized. Bovin Serum Albumin (BSA) was used to block unspecific sites with a concentration of $0.1 \%$ in PBS buffer.

2.2. T4-Phage Immobilization and Bacteria Culture. The physisorption of T4-phage on gold surface and bacteria culture was reported in previous work [18]. For biosensor application, all measurements were performed at a room temperature of 27 degrees.

2.3. SPR Instrumentation. Work was carried using the SPR imager II (from GWC Technologies, USA), which is designed for analysis of molecular interactions in an array format with 16 spots. The entire microarray was exposed to the same analyte using the $1 \mathrm{~mL}$ flow cell. After the self-assembly, the 16-spot microarray is divided in two independent compartments by a polydimethylsiloxane (PDMS) gasket in the microfluidic chamber. The CCD camera simultaneously captures all data for all probes on the microarray (Figure 1). The chips were washed with ethanol and inserted into the SPRi and all measurements were performed with sterile PBS buffer.

2.4. Refractive Index and Temperature Effects. The SPRi was initially calibrated versus different percentages of triethylene glycol dissolved in ultrapure water. All solutions were stirred for ten minutes and refractive index was measured with a digital Abbe refractometer in parallel. In order to study the effect of temperature in the measurement, the SPRi curves were obtained for different temperature values in the range of 24 to 40 degrees.

\section{Results and Discussions}

3.1. SPR Imager Calibration. The chip was inserted into the SPRi placed under a flux of ultrapure water, then under a flux of ultrapure water mixed with different percentages of triethylene glycol. Figure 1(a) shows a calibration curve which presents variation in pixels over time for different percentages of triethylene glycol in ultrapure water at fixed

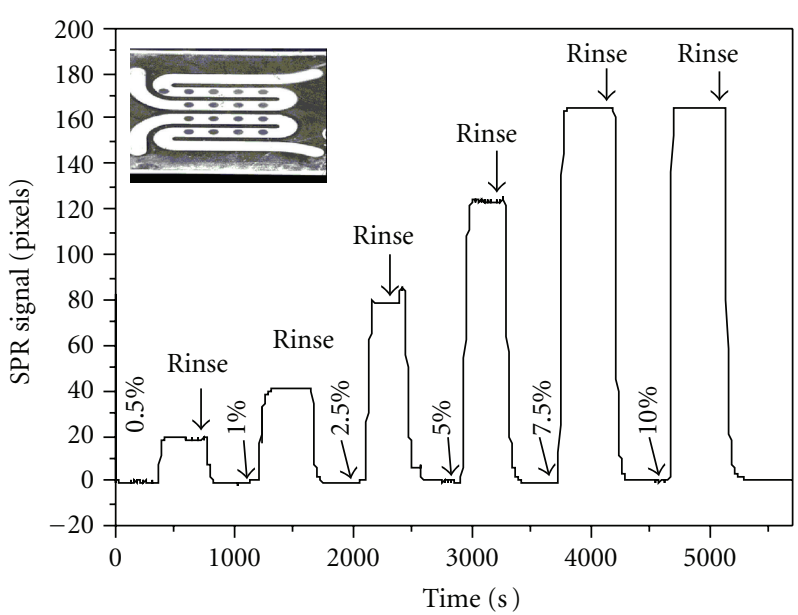

(a)

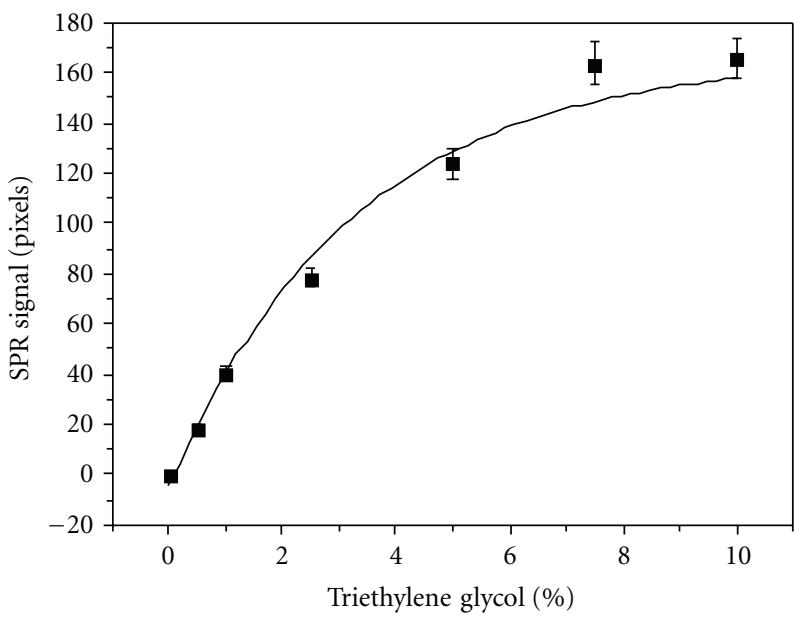

(b)

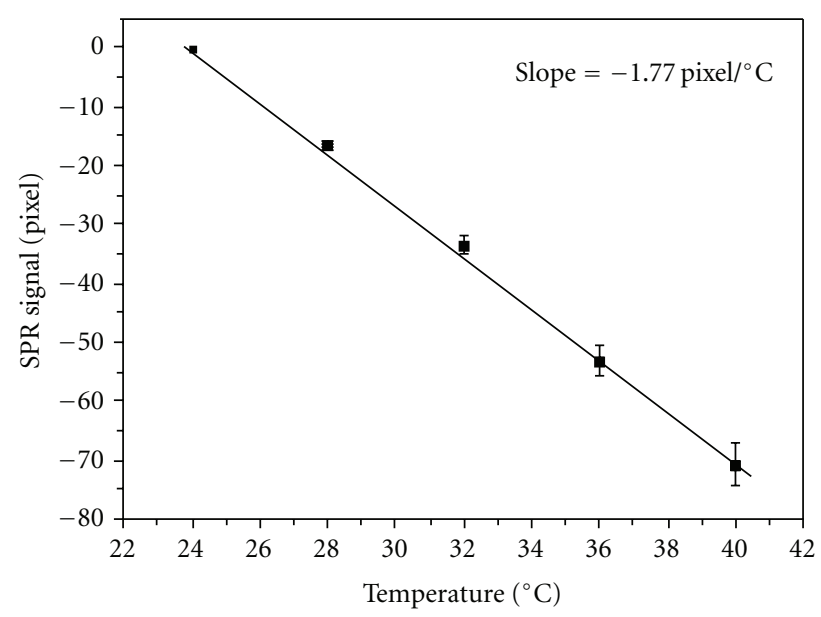

(c)

FIGURE 1: (a) SPRi signal over time for different percentages of triethylene glycol in ultrapure water at fixed temperature (27 degrees). (b) Calibration curve of SPRi signal versus different percentage of triethylene glycol in ultrapure water. (c) SPRi signal versus temperature variation. 
TABLE 1: SPRi signal and refractive index of the external medium obtained with different percentage of triethylene glycol per $1 \mathrm{~mL}$ of ultrapure water.

\begin{tabular}{lccccccc}
\hline \% Triethylene glycol in ultrapure water & 0 & 0.5 & 1 & 2.5 & 5 & 7.5 & 10 \\
SPR signal (pixels) & 0 & 18.86 & 40.69 & 78.35 & 124 & 164.63 & 164.63 \\
Refractive Index & 1.33300 & 1.33305 & 1.33410 & 1.33620 & 1.33945 & 1.34180 & 1.34360 \\
\hline
\end{tabular}
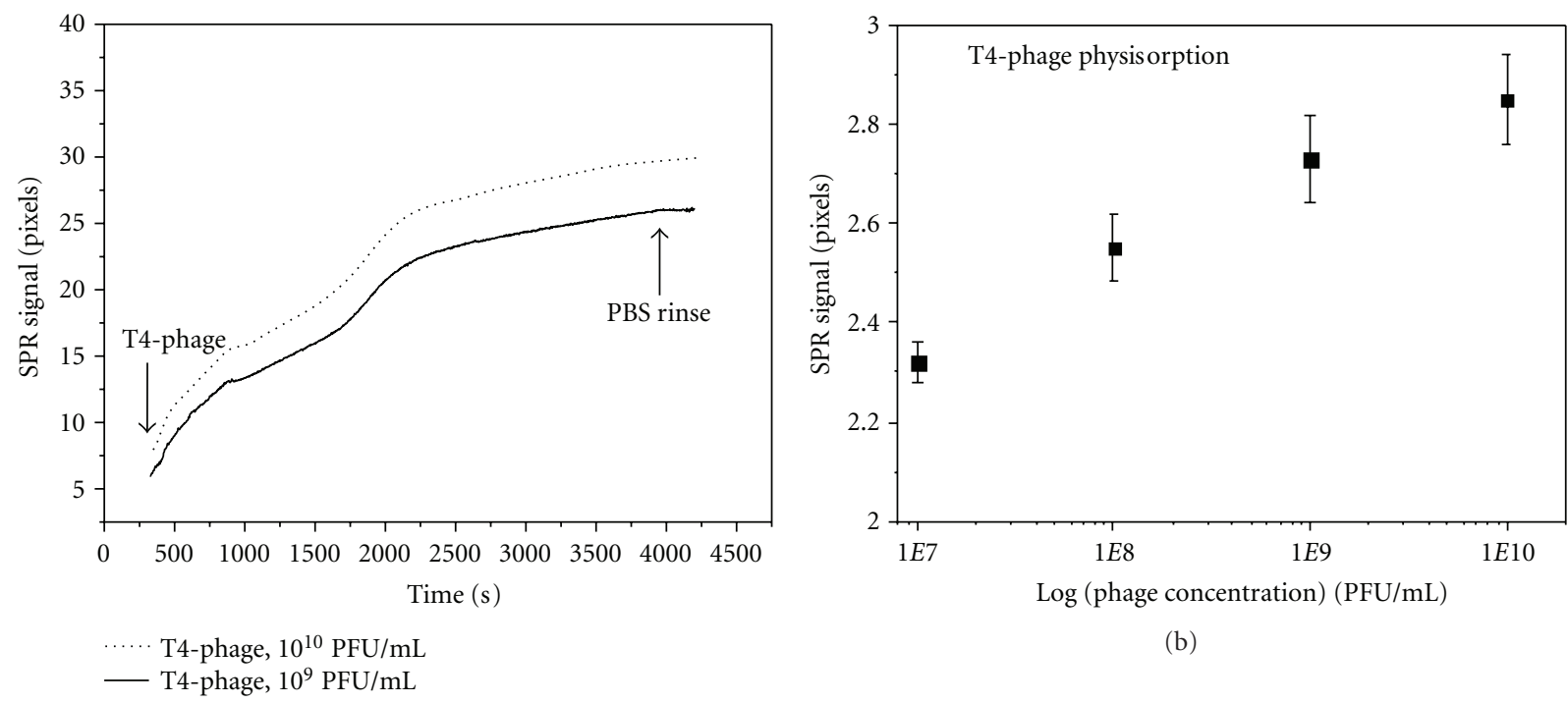

(b)

(a)

FIGURE 2: (a) SPRi signal over time for different T4-phage concentration physisorbed on the 16 gold spots. (b) Calibration curve of SPRi signal versus different concentration of T4-phages physisorbed on the 16 gold spots.

temperature (27 degrees). The variation in signal registered by the CCD camera is due to the increase of the refractive index of the external medium. A commercial digital refractometer was used to determine the index values of each percentage (Table 1). A variation of $5 \times 10^{-5}$ in refractive index can be easily detected, which induces a 20-pixel variation. Figure 1 (b) shows the calibration curve of SPR signal variation for different percentages of triethylene glycol in ultrapure water. As it can be seen, the SPR signal increases for increasing the refractive index of the external medium. Signal saturation was reached at $10 \%$ of triethylene glycol in ultrapure water. The noise signal was equal to 1 pixel and the SPRi system can detect a refractive index variation below $5 \times 10^{-5}$ which is very interesting for biochemical detection. Figure 1(c) shows the CCD camera signal variation versus temperature effect. The slope shows a shift of $1.77 \mathrm{pixel} /$ degrees which is due to refractive index medium changes. For biosensors application, the temperature will be fixed at 27 degrees.

3.2. T4-Phage Deposition. Figure 2(a) shows the pixels variation over time for T4-phage physisorption on the spots. The adsorption of phages induces a signal variation due to thin layer deposition on gold spots. Measurements were carried out for different phage concentration in order to optimize surface coverage (Figure 2(b)). As illustrated in Figure 2(b), signal increases over time and with phage concentration which is due to higher surface covering efficiency.

Physisorption of T4-phages on gold surface was additionally confirmed by obtaining Atomic Force Microscopy (AFM) images in tapping modes. Figure 3 shows the AFM images of the gold chips after physisorption of T4-phages. As it can be observed, the structure of gold surface can be distinguished clearly from phages. In this respect, phages were organized in aggregate formats over the whole surface, a behaviour that has been previously observed [19].

3.3. Detection and Selectivity. Figure 4(a) shows the CCD camera signal variation over time for immobilized T4-phage before and after injection of $10^{4} \mathrm{CFU} / \mathrm{mL}$ E. coli (gram negative) with BSA as blocking layer. The increase in the signal is due to the specific recognition of bacteria by the T4-phage. The same behaviour was observed in the other eight gold spots of the channel, all of them modified with T4-phage and exposed to E. coli. A small perturbation was observed after 30 minutes which can be due to the lysis of bacteria. The lysis of bacteria can induce a small variation in refractive index which cannot be observed clearly with the SPR technique [20]. Different concentrations of E. coli and Lactobacillus ranging from $10^{4} \mathrm{CFU} / \mathrm{mL}$ to $10^{7} \mathrm{CFU} / \mathrm{mL}$ were 


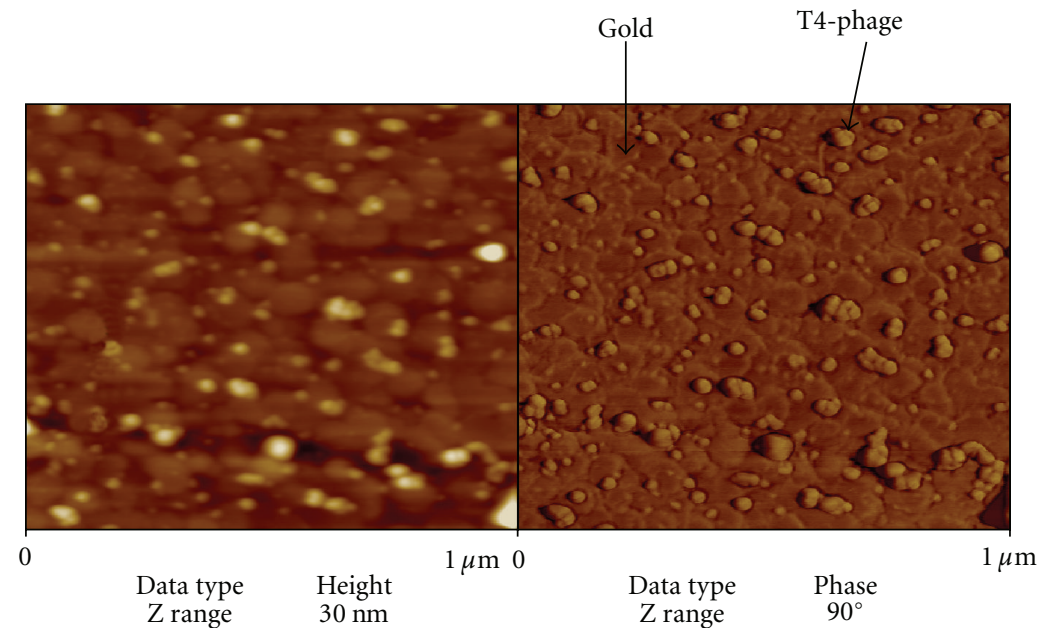

Figure 3: AFM images of T4-phage deposited on gold surface.

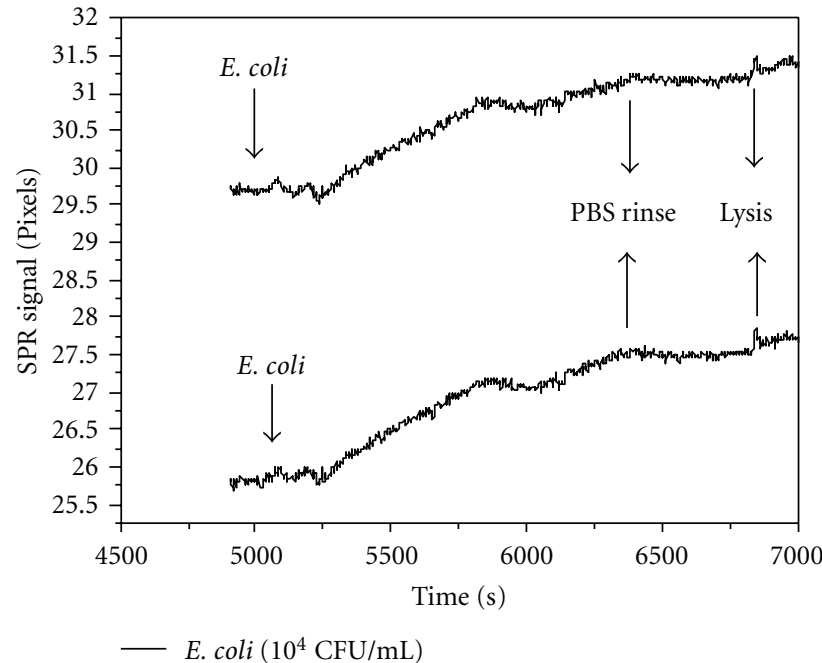

(a)

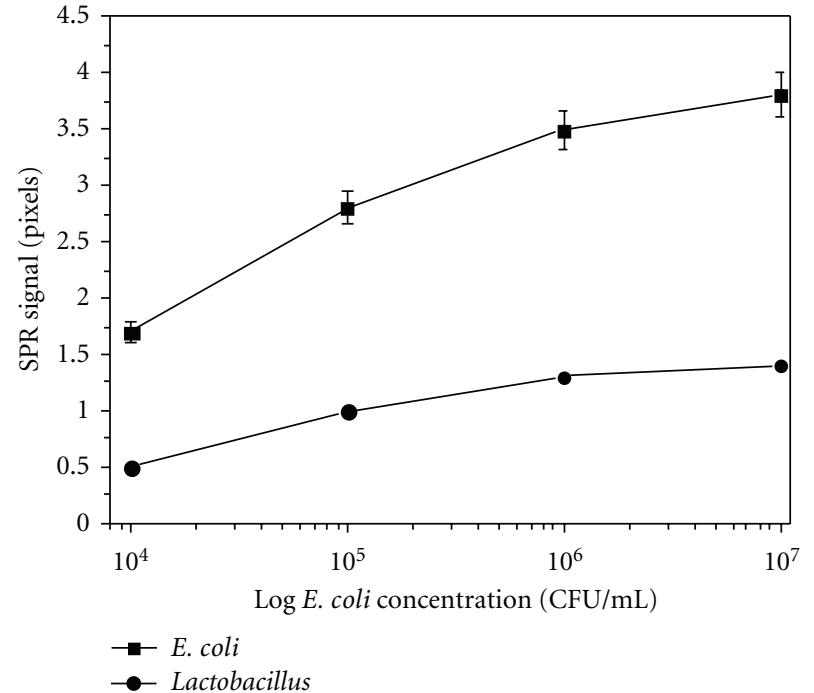

(b)

Figure 4: (a) SPRi signal over time after bacteria injection $\left(10^{4} \mathrm{CFU} / \mathrm{mL}\right.$ of E. coli). (b) Calibration curve of SPRi signal versus E. coli concentrations (squares) and versus Lactobacillus bacteria (circle).

finally tested using T4-phage immobilized on gold arrays. Figure 4(b) summarizes the SPR signals registered versus E. coli concentrations. The increase of E. coli concentration leads to an increase of SPR signal, due to the successful recognition of bacteria by the virus. A detection limit of $10^{4} \mathrm{CFU} / \mathrm{mL}$ E. coli concentrations was obtained with T4phages with a good reproducibility. According to the noise of 1 pixel, detection limit below $10^{4} \mathrm{CFU} / \mathrm{mL}$ can be detected for E. coli better than those obtained with others electrochemical, SPR, and Surface Acoustic Wave (SAW) techniques [18, 21-23]. A negative control was performed by injecting Lactobacillus bacteria, as gram-positive bacteria that is not recognized by T4-phage. A very low signal has been obtained compared to the specific signal (Figure 4(b)).

\section{Conclusion}

In this work, we studied the sensitivity of an SPRi to refractive index and temperature variation. A new SPR imager biosensor based on T4-phage immobilized on gold surfaces by physisorption technique was developed. The SPRi biosensor was used for E. coli detection using Lactobacillus bacteria as a negative control. The results show selectivity of the T4phages modified surface and a good sensor reproducibility. 
A detection limit below $10^{4} \mathrm{CFU} / \mathrm{mL}$ can be detected for $E$. coli better than those obtained with other techniques. For future work, detection limit will be improved and other phages will be used.

\section{Acknowledgments}

Professor A. Abdelghani thanks the NATO (North Atlantic Treaty Organization) for the material donation. The authors thank Biophage Pharma (Canada) for providing them T4phage, Dr. Mohamed Zourob (Canada), Professor A. Simonian (USA), Dr. Eva Baldrich (CNM Barcelona, Spain), and F. J. Del Campo (CNM Barcelona, Spain) for their collaboration.

\section{References}

[1] H. S. Jang, K. N. Park, C. D. Kang, J. P. Kim, S. J. Sim, and K. S. Lee, "Optical fiber SPR biosensor with sandwich assay for the detection of prostate specific antigen," Optics Communications, vol. 282, no. 14, pp. 2827-2830, 2009.

[2] D. A. Healy, C. J. Hayes, P. Leonard, L. McKenna, and R. O'Kennedy, "Biosensor developments: application to prostatespecific antigen detection," Trends in Biotechnology, vol. 25, no. 3, pp. 125-131, 2007.

[3] Y. Wang, A. Brunsen, U. Jonas, J. Dostálek, and W. Knoll, "Prostate specific antigen biosensor based on long range surface plasmon-enhanced fluorescence spectroscopy and dextran hydrogel binding matrix," Analytical Chemistry, vol. 81, no. 23, pp. 9625-9632, 2009.

[4] C. Cao, J. P. Kim, B. W. Kim et al., "A strategy for sensitivity and specificity enhancements in prostate specific antigen$\alpha 1$-antichymotrypsin detection based on surface plasmon resonance," Biosensors and Bioelectronics, vol. 21, no. 11, pp. 2106-2113, 2006.

[5] W. P. Hu, H. Y. Hsu, A. Chiou et al., "Immunodetection of pentamer and modified C-reactive protein using surface plasmon resonance biosensing," Biosensors and Bioelectronics, vol. 21, no. 8, pp. 1631-1637, 2006.

[6] S. F. Chou, W. L. Hsu, J. M. Hwang, and C. Y. Chen, "Development of an immunosensor for human ferritin, a nonspecific tumor marker, based on surface plasmon resonance," Biosensors and Bioelectronics, vol. 19, no. 9, pp. 999-1005, 2004.

[7] C. Corne, J. B. Fiche, D. Gasparutto et al., "SPR imaging for label-free multiplexed analyses of DNA N-glycosylase interactions with damaged DNA duplexes," Analyst, vol. 133, no. 8, pp. 1036-1045, 2008.

[8] N. Bassil, E. Maillart, M. Canva et al., "One hundred spots parallel monitoring of DNA interactions by SPR imaging of polymer-functionalized surfaces applied to the detection of cystic fibrosis mutations," Sensors and Actuators, B, vol. 94, no. 3, pp. 313-323, 2003.

[9] B. Barlen, S. D. Mazumdar, O. Lezrich, P. Kämpfer, and M. Keusgen, "Detection of salmonella by surface plasmon resonance," Sensors, vol. 7, no. 8, pp. 1427-1446, 2007.

[10] B. K. Oh, W. Lee, Y. K. Kim, W. H. Lee, and J. W. Choi, "Surface plasmon resonance immunosensor using self-assembled protein G for the detection of Salmonella paratyphi," Journal of Biotechnology, vol. 111, no. 1, pp. 1-8, 2004.
[11] H. Baccar, M. B. Mejri, I. Hafaiedh, T. Ktari, M. Aouni, and A. Abdelghani, "Surface plasmon resonance immunosensor for bacteria detection," Talanta, vol. 82, no. 2, pp. 810-814, 2010.

[12] A. Abdelghani, C. Veillas, J. M. Chovelon, N. JaffrezicRenault, and H. Gagnaire, "Stabilization of a surface plasmon resonance (SPR) optical fibre sensor with an ultra-thin organic film: application to the detection of chloro-fluoro-carbon (CFC)," Synthetic Metals, vol. 90, no. 3, pp. 193-198, 1997.

[13] S. Herminjard, Mid-infrared surface plasmon resonance sensing applied to refractive index measurements of liquids, Ph.D. thesis, EPFL, Lausanne, Switzerland, 2010, Thesis no. 4603.

[14] K. S. Lee, J. M. Son, D. Y. Jeong, T. S. Lee, and W. M. Kim, "Resolution enhancement in surface plasmon resonance sensor based on waveguide coupled mode by combining a bimetallic approach," Sensors, vol. 10, no. 12, pp. 1139011399, 2010.

[15] GWC Technologies Inc., Technical Note 122, Madison, USA.

[16] J. M. Brockman, B. P. Nelson, and R. M. Corn, "Surface plasmon resonance imaging measurements of ultrathin organic films," Annual Review of Physical Chemistry, vol. 51, pp. 4163, 2000.

[17] A. F. González, J. Rychłowska, R. Badía, and R. Salzer, "SPR imaging as a tool for detecting mucin-anti-mucin interaction. Outline of the development of a sensor for nearpatient testing for mucin," Microchimica Acta, vol. 158, no. 34, pp. 219-225, 2007.

[18] M. B. Mejri, H. Baccar, E. Baldrich et al., "Impedance biosensing using phages for bacteria detection: generation of dual signals as the clue for in-chip assay confirmation," Biosensors and Bioelectronics, vol. 26, no. 4, pp. 1261-1267, 2010.

[19] B. Aoune, D. Neff, and M. Norton, Chemistry Master of Science Program Review, M.S. thesis, Marshall University, Huntington, WVa, USA, 2006.

[20] T. A. T. Tran, Bacteriophage T4 Lysis and Lysis Inhibition: Molecular Basis of an Ancient Story, Texas A\&M University, College Station, Tex, USA, 2007.

[21] A. Shabani, M. Zourob, B. Allain, C. A. Marquette, M. F. Lawrence, and R. Mandeville, "Bacteriophage-modified microarrays for the direct impedimetric detection of bacteria," Analytical Chemistry, vol. 80, no. 24, pp. 9475-9482, 2008.

[22] C. A. Meeusen, E. C. Alocilja, and W. N. Osburn, "Detection of E.coli O157:H7 using a miniaturized surface plasmon resonance biosensor," Transactions of the American Society of Agricultural Engineers, vol. 48, no. 6, pp. 2409-2416, 2005.

[23] N. Moll, E. Pascal, D. H. Dinh et al., "A Love wave immunosensor for whole E.coli bacteria detection using an innovative twostep immobilisation approach," Biosensors and Bioelectronics, vol. 22, no. 9-10, pp. 2145-2150, 2007. 


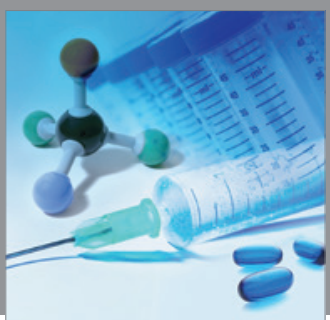

International Journal of

Medicinal Chemistry

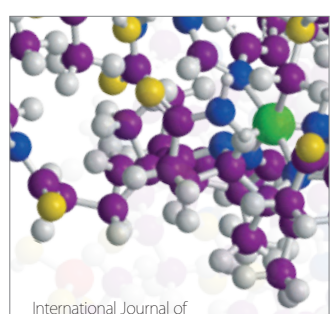

Carbohydrate Chemistry

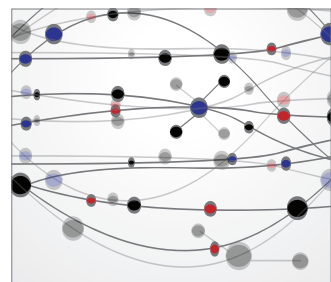

The Scientific World Journal
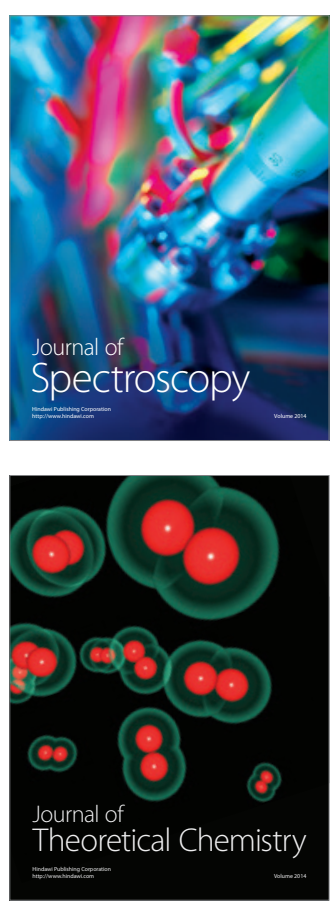
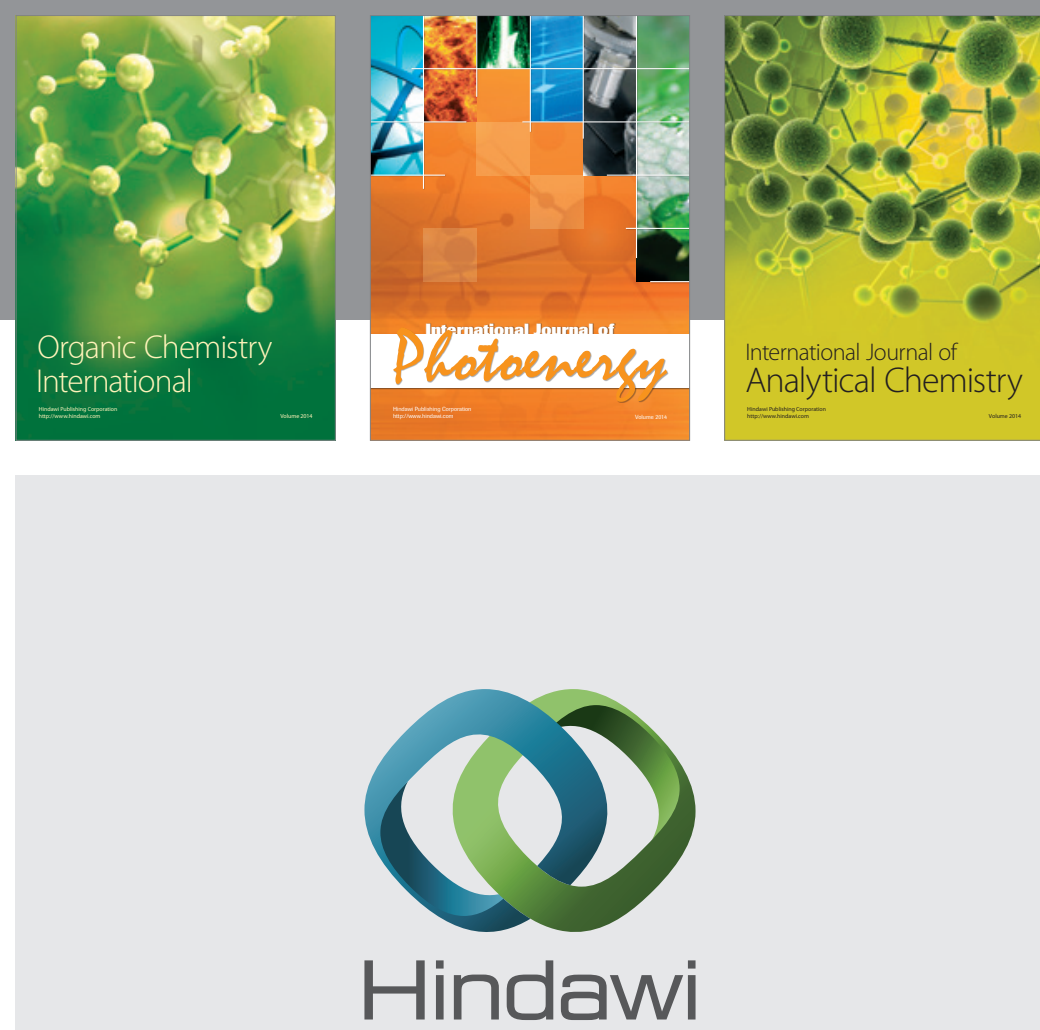

Submit your manuscripts at

http://www.hindawi.com
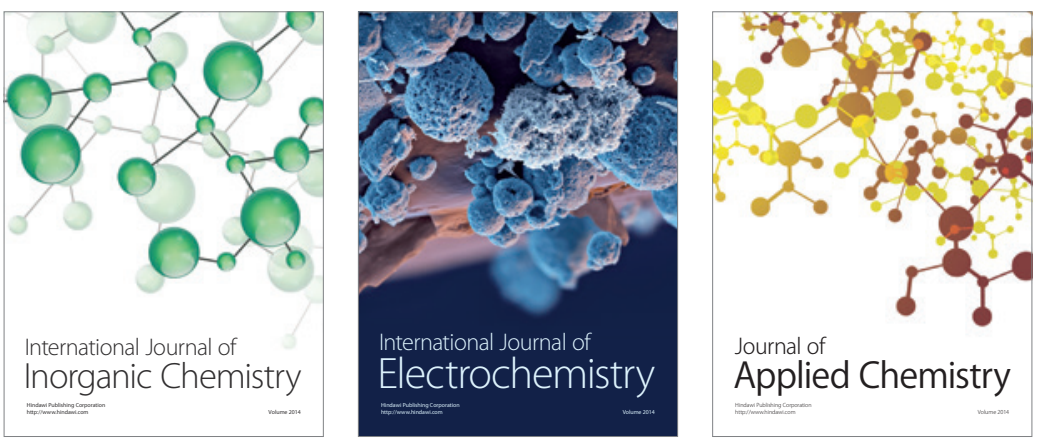

Journal of

Applied Chemistry
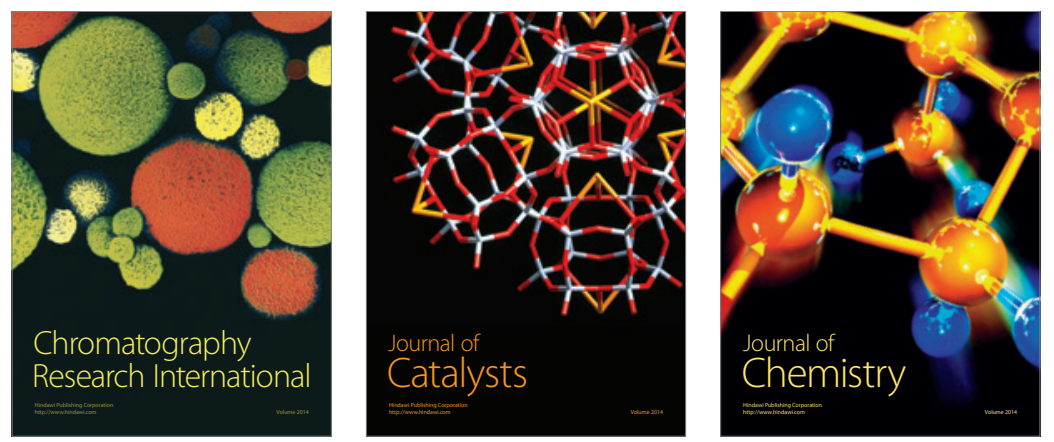
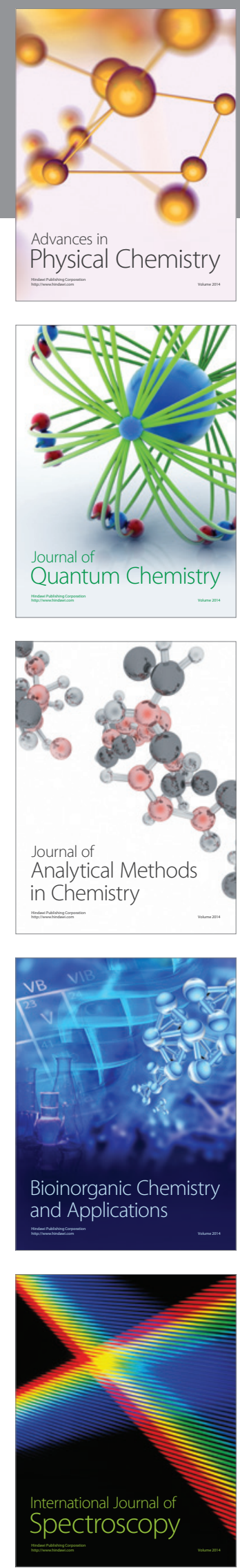